\title{
Enhancing the QoS of Integrated Mobile Ad hoc Network using Multiple Cluster Head Gateway
}

\author{
Ashish Bagwari \\ IEEE Member and Research Scholar \\ Electronics and Communication \\ Dehradun Institute of Technology, \\ Dehradun, India
}

\author{
Sourabh Bisht \\ Research Scholar \\ Electronics and Communication \\ Graphic Era University, \\ Dehradun, India
}

\begin{abstract}
Mobile Ad Hoc Network (MANET) consists of dynamic topology as nodes in the network are mobile, and connected via wireless links. Nodes within the cluster communicate directly. However, nodes communicate outside the cluster through a centralized node that is called a Cluster Head Gateway (CHG) [1]. As the number of nodes increases complexity of MANET increases in various issues. Ad hoc wireless network is a dynamic multi-hop network, which is established by a group of mobile nodes on a shared wireless channel. The shared medium and the multi-hop nature of the wireless ad hoc networks pose fundamental challenges to the design of an effective resource allocation algorithm to maximize the aggregated utility of flows, maintaining basic fairness among the multiple flows. An elected Cluster Head Gateway (CHG) is assigned for communication with all other clusters. The centralized Cluster Head Gateway can become a bottleneck and possibly cause a lower connectivity for the clustering system. In this paper we propose a mechanism in which communication outside the cluster is distributed through separate Cluster Head Gateways in order to enhance the QoS of MANET. The CHGs should provide better QoS to/than other MANET nodes within network. Now here the QoS deals with several parameters like Throughput, End-End Delay, Traffic Sent and Traffic Received. We also show that the increase in overall QoS and connectivity is by the increase/add Cluster Head Gateways. Additionally, the routing reliability to outside the cluster is increased since routes to different clusters use distinct Cluster Head Gateways. Finally, this paper conducts simulation experiments in the conditions where we using Multiple CHG to the nodes within a network.
\end{abstract}

\section{Keywords}

Cluster Head Gateway, Throughput, MANET, Multiple Cluster Head Gateway, QoS, End-End Delay.

\section{INTRODUCTION}

A wireless ad hoc network consists of mobile nodes that move freely and communicate with each other using wireless links. A MANET system does not require a predetermined infrastructure and nodes act as hosts and as routers for other nodes. Nodes are grouped into distinct or overlapping clusters. Clustering provides a Hierarchical MANET system which assists in making the routing scalable. Some of the nodes are elected to be part of the backbone for the MANET system [2] [3] [10] [12-14]. These nodes are called Cluster Head Gateway. Cluster Head Gateways are elected according to several techniques [1]. The Cluster
Head Gateway allows for minimizing routing details overhead from other nodes within the cluster. MANET requires efficient routing algorithm in order to reduce the amount of signaling introduced due to maintaining valid routes[4][5], and therefore enhance the overall performance of the MANET system. As the Cluster Head Gateway is the central node of routing for packets destined outside the cluster in the distinct clustering configuration, the Cluster Head Gateway computing machine pays a penalty of unfair resource utilization such as battery [15], CPU, and memory. As we can say using Multiple Cluster Head Gateway we distribute the load among multiple hosts in the cluster [1] and gets better QoS i.e. having high throughput, less End-End delay and low packet drops.

The rest of the paper is organized as follows: Section II presents the related work. Section III describes our proposed working model. Section IV presents the simulation experiment setup and gives the performance evaluation of our proposed strategy. Section V concludes the paper.

\section{RELATED WORK}

Several mechanisms of Cluster Head election exist with an objective to provide stable and efficient routing in the MANET system [4] [8] [13-14]. Some mechanism assigns the Cluster Head based on the node id as in the Linked Cluster Algorithm (LCA) which selects as the Cluster Head the node with the highest ID [3]. Other mechanisms favor allowing some type of fair share of Cluster Head responsibility by changing the Cluster Head based on an assigned ID to the Cluster Head [2]. A node with a high mobility rate is higher than the duration of Cluster Head rotation may not get the chance to become a Cluster Head. Other Cluster Head election mechanisms consider relative node mobility to ensure routing path availability [3] [9] [11]. However, causing an added signaling overload and causing the elected Cluster Head to pay the higher resource utilization penalty. We can conclude from the existing research that several tradeoffs exist for the elected Cluster Head and the other cluster nodes.

- Firstly, the Cluster Head has to bear higher resource utilization such as power, which may deplete its battery sooner than other nodes in the cluster.

- Secondly, despite fair share responsibility of Cluster Head role, it is possible that heavy burst of traffic takes place causing some Cluster Heads to use maximum resources. 
- Thirdly, the fair share or load balancing technique [2], might result in a Cluster Head that will not provide the optimal path for routing, or yet a link breakage.

There is no one common Cluster Head election mechanism that is best for MANET systems, without some tradeoffs.

The Zone Routing Protocol, ZRP, in [7], provides a hybrid approach which produces added routing control messages in the network due to keeping up to date routes [7]. ZRP divides the network into overlapping zones, while clustering can have distinct, non overlapping clusters. In addition, the authors in [67] claim this hybrid approach is suited for large networks, enhances the system throughput, but adds more complexity. As discussed above, the main focus of the previous work focuses on an election of one Cluster Head for a cluster. Even though this minimizes the overall signaling overhead in the cluster, but it mainly can make the central Cluster Head a bottleneck.

\section{PROPOSED WORKING MODEL}

The base of our Paper is enhance the QoS of MANET via distributing the load of the Cluster Head Gateway amongst multiple Cluster Head Gateways in the same cluster and also provides better connectivity between nodes as shown in figure 2 , in the architecture of multiple Cluster Head Gateways, one CHG for each neighboring clusters. Any of the prior work can be used to select the Cluster Head Gateways for a cluster. In the case of one Cluster Head Gateway per cluster, a link breakage caused by the failure of the Cluster Head Gateway isolates all cluster nodes from communicating to/from outside the cluster. However, our approach reduces the link breakage to be only in the direction towards a path where the failed Cluster Head Gateway forwards the data. Therefore, the reliability of routing in the MANET system is increased. Our architecture does not state the routing protocol as in [7], but leaves the decision as done in the original designs of clustering [2] [3][10][12-14]. Our MANET system consists of 4 distinct non-overlapping clusters as shown in figure 1 .

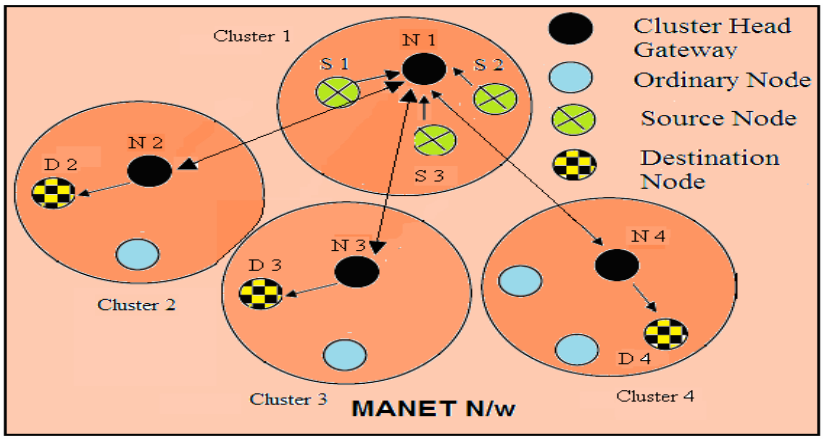

Figure 1. Showing the Previous Clustering Approach

As shown in figure 1 Cluster 1, 2, $3 \& 4$ all have one Cluster Head Gateway. Our traffic included FTP traffic generated between nodes in cluster 1 and nodes in all other clusters in the MANET system. In order to focus on the objective of distributing the Cluster Head Gateway load. Here we have one Cluster Head Gateway in cluster 1, to send a packet from a node $\mathrm{S} 1$ in cluster 1 to a node $\mathrm{D} 2$ in cluster 2 , the packet is routed via the single Cluster Head Gateway N1 in cluster 1 and finally via the Cluster Head Gateway N2 in cluster 2 to cluster node D2. Then routing from cluster 1 to cluster 3 was from cluster node
S2 to Cluster Head Gateway N1 in cluster 1 passes to the Cluster Head Gateway N3 in cluster 3 to cluster node D3.

However, routing from cluster 1 to cluster 4 was also done directly from cluster node S3 to Cluster Head Gateway N1 in cluster 1 passes to the Cluster Head Gateway N4 in cluster 4 to cluster node D4.

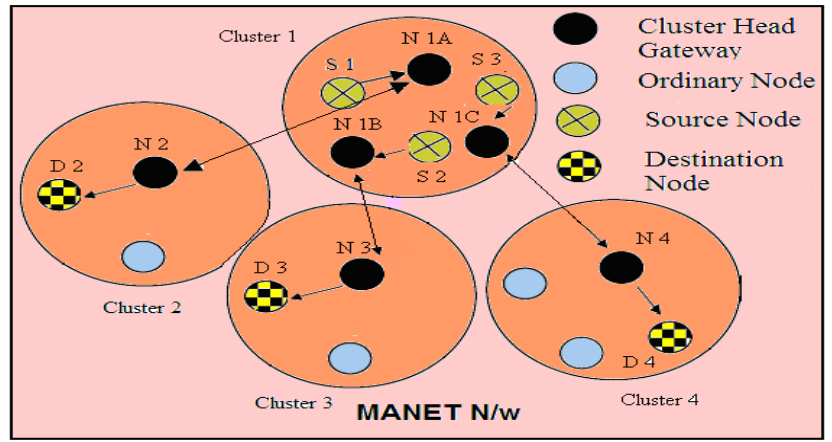

Figure 2. Showing the New Clustering Approach

As shown in figure 2, Cluster 1 is the one which we had as a cluster with multiple Cluster Heads Gateway. The remaining clusters 2, 3 and 4 operated with one Cluster Head Gateway. When using multiple Cluster Head Gateways in cluster 1, a different Cluster Head Gateway was used for routing to each of the neighboring clusters. For example, routing from cluster 1 to cluster 2 uses a Cluster Head Gateway N1A that is different when routing from cluster 1 to cluster $3 \&$ cluster 4 use N1B \& N1C respectively. Therefore, since there are 3 neighboring clusters to cluster 1 , the system allowed for the use of 3 Cluster Head Gateways, one for routing to/from each neighboring cluster.

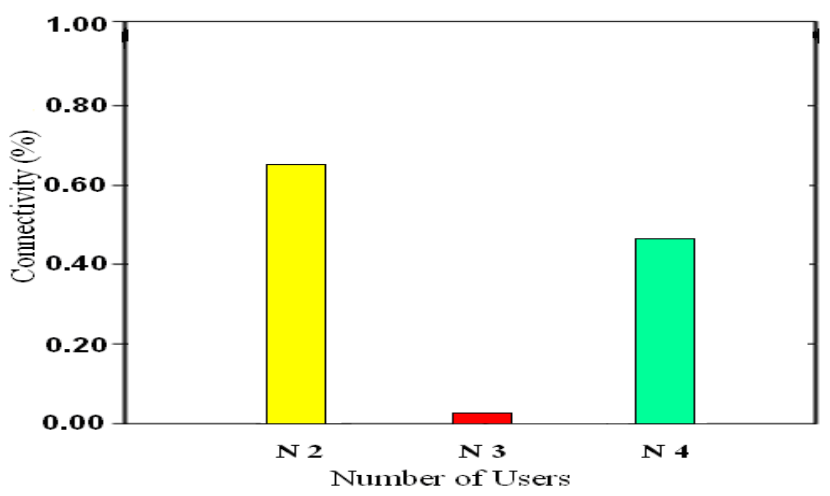

Figure 3. Connectivity (\%) Vs No. of Users using Single CHG

In figure 3 showing the Connectivity Vs No. of Users graph for figure1 Suppose the links between N1 \& N2, N1 \& N4 are ok but N1 to N3 is break due to the more traffic occurrence and occurred traffic is not handled by assign Band width of this link (N1 \& N3). Due to this Connectivity of this link reduce or approximate zero using the Single Cluster Head Gateways. And suppose CHG of cluster-1 (N1) fails then all the communication with other CHG's via N1 will break like S1-D2, S2-D3 \& S3D4. This previous MANET system approach is not capable of 
handling more No. of users if traffic increases and also not providing better connectivity if CHG like $\mathrm{N} 1$ for cluster-1 fails. which is shown the lower Connectivity when using single Cluster Head Gateways in given graph i.e. the percentage of Connectivity for the Node N2 and N4 at about $62 \%$ and $44 \%$ respectively While for Node $\mathrm{N} 3$ is $2 \%$ which is near about $0 \%$ due to breakage.

In our topology figure4 showing the Connectivity Vs No. of Users graph taking figure2 under consideration where links between N1A to N2, N1B to N3 \& N1C to N4 are OK via using different CHG's (N1A, N1B \& N1C). We increased the number of Cluster Head Gateways to 3 i.e. if any CHG fail it will not effect other CHG communication due to using multiple CHG. However, our Connectivity is increasing as shown in figure4. In addition, as the traffic arrival rate increased due to having the 3 Cluster Head Gateways, the service rate also increased, resulting in the same utilization rate for the MANET system. Therefore, we conclude that the number of Cluster Head Gateways increase should improve connectivity by a factor equals to the number of added Cluster Head Gateways. The connectivity results are presented in figure 4 . The results show the percentage of increase in the averaged cumulative connectivity for running multiple Cluster Head Gateways over one Cluster Head Gateway. We have noticed, as shown in figure4, the percentage of Connectivity improvement for the Node N2 at about $72 \%$ \& Node N3 is $20 \%$ While for Node N4 near about $50 \%$.

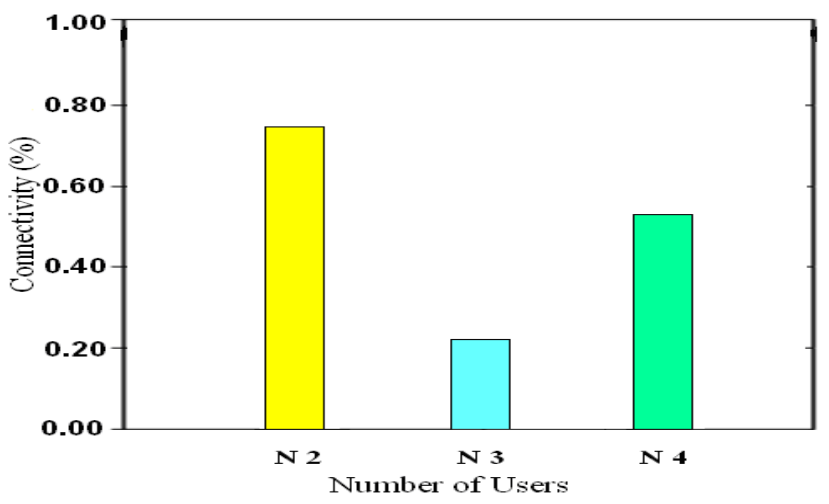

Figure 4. Connectivity (\%) Vs No. of Users using Multiple CHG

So after analyzed all results we can say

- (Connectivity) $\propto$ (No. of Cluster Head Gateway).

\section{SIMULATION SETUP AND RESULTS DISCUSSION}

\subsection{Simulation Setup}

To simulate our Cluster Head Gateway Network, we used Opnet $14.0 \mathrm{v}$. The simulation parameters and their values are given in Table 1.
Table 1. Simulation Parameters

\begin{tabular}{|l|l|}
\hline Parameter & Value \\
\hline Number of MANET Nodes & 30 \\
\hline $\begin{array}{l}\text { Number of Packet (Traffic) } \\
\text { Sources }\end{array}$ & 4 \\
\hline $\begin{array}{l}\text { Number of Cluster Head } \\
\text { Gateway (CHG) }\end{array}$ & 15 \\
\hline Size of Area & $1000 * 1000$ (m.) \\
\hline Transmission Range & 250 (m.) \\
\hline Traffic Type & Constant Bit Rate (CBR) \\
\hline Standard Ad hoc Speed & $20 \mathrm{~m} / \mathrm{s}$ \\
\hline Datagram forwarding rate & 100,000 (packets/sec) \\
\hline Simulation Time & 900 (sec) \\
\hline Wireless Channel Bandwidth & 1000 (KHz) \\
\hline Beacon Interval & 0.02 (sec) \\
\hline Node Movement Model & 500,000 (packets/sec) \\
\hline Dataity (RPGM)
\end{tabular}

\subsection{Results Discussion}

The Performance of the proposed CHG N/w is analyzed with respect to Throughput, End-End Delay, Traffic Sent and Traffic Received.

Figure 5 to 8 shows the performance with respect to the throughput, end-end delay, traffic sent and traffic received respectively. The performance is first evaluated by $\mathrm{CHG}$ connectivity.

Figure 5 showing the Throughput of the whole network with respect to time, Firstly when time period is $2 \mathrm{~min}$, throughput is $7,000,000$ (bits/sec) which is maximum value, after increasing the time period throughput is almost constant and varying across 7,000,000 (bits/sec) till $15 \mathrm{~min}$. time period. It also shows the better throughput along with better connectivity between the nodes. 


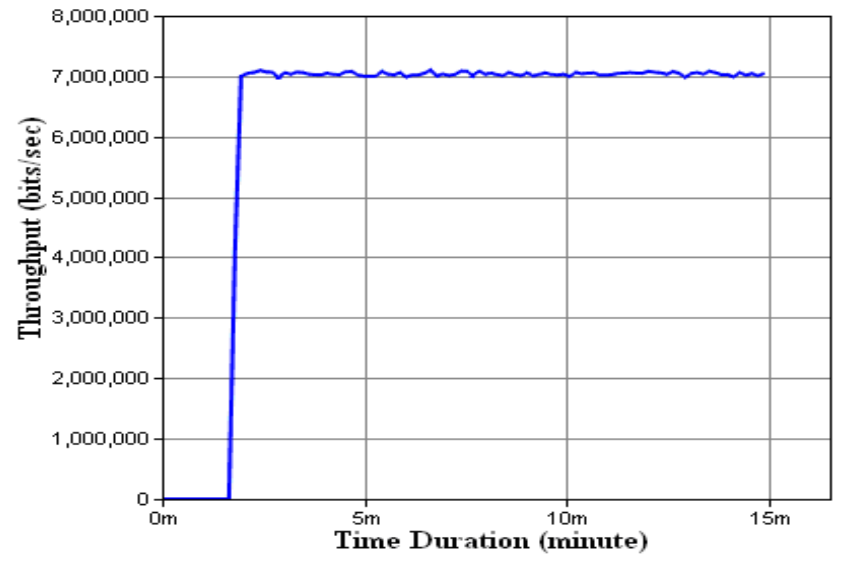

Figure 5. Throughput Vs Time Duration

Figure 6 showing End-End Delay Vs time Duration graph. The End-End Delay is 0.039 (sec) at 2 min. after that it is drastically increasing up to $0.10(\mathrm{sec})$ and then constantly varying across 0.10 (sec) up to $15 \mathrm{~min}$ time period. End-End Delay is less due to using Multiple Cluster Head Gateways.

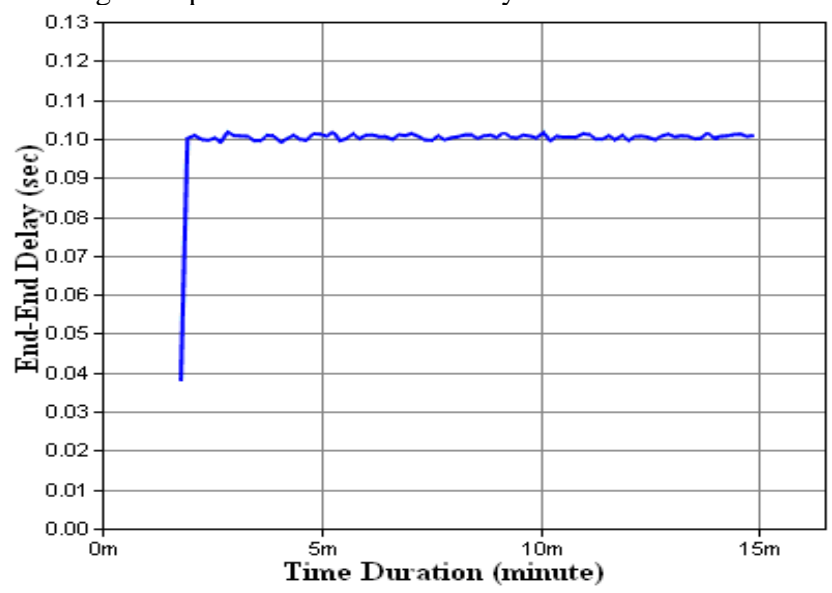

Figure 6. End-End Delay Vs Time Duration

Figure 7 shows Traffic Sent Vs time Duration graph. Here Traffic sent maximum value is 80 (packets/sec) at 2 min. time period. When time period is increasing the value of Traffic Sent is constant varying across 80 (packets/sec) till $15 \mathrm{~min}$ time period.

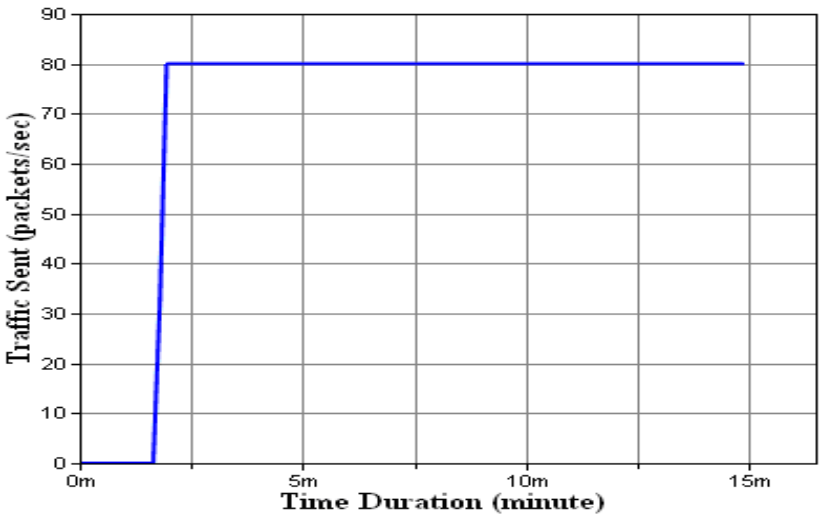

Figure 7. Traffic Sent Vs Time Duration
Figure 8 shows Traffic Received Vs time Duration graph. Here Traffic received value is varying between 24 (packets/sec) and 28 (packets/sec) up to $15 \mathrm{~min}$. time period.

With the help of Figure 7 and 8 we can analyze the graph between Traffic Sent and Traffic Received. And also calculate the data loss (packet drops).

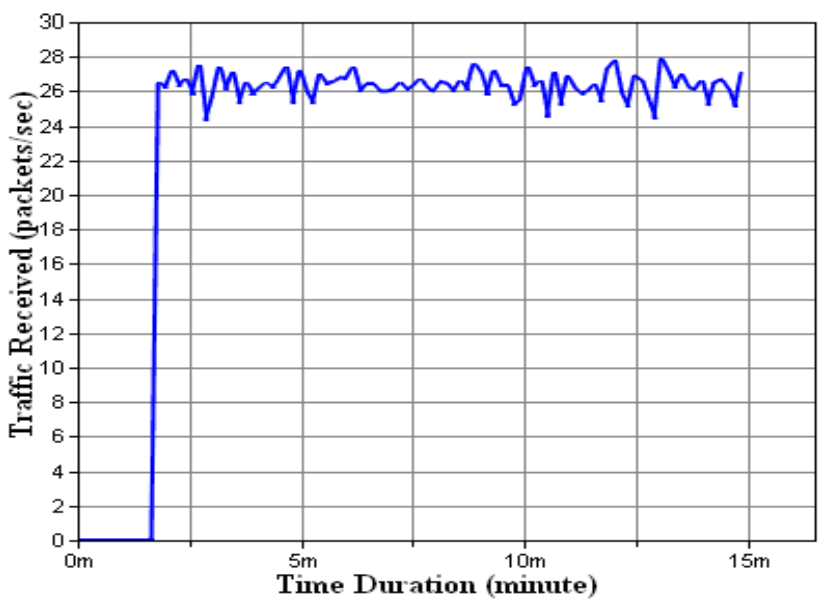

Figure 8. Traffic Received Vs Time Duration

\section{CONCLUSION}

In this paper, we have proposed enhancing the QoS of Integrated Mobile Ad hoc Network using Multiple Cluster Head Gateway. Where QoS dealing with throughput, end-end delay and packet drops (calculated with the help of sent packet and received packet). One of the distinguishing characteristics of our strategy is that, the MANET node is provided a better Quality of Service and connectivity with better throughput, minimum End-End Delay and packet drops using multiple CHG. In addition, routing reliability is increased since a failure of one Cluster Head Gateway does not break all routing to outside the cluster. A detailed performance was made with respect to Throughput, End-End Delay, Traffic Sent and Traffic Received. The simulation results confirm that, the purposed approach has better connectivity, good throughput, less End-End Delay and data loss. Therefore it can be used to extend the network coverage. In future the $\mathrm{CHG}$ approach can be evaluated under different mobility scenarios and the performance can be measured with other large N/w's. As wireless communication becomes increasingly prevent, $\mathrm{CHG}$ approach is becoming a viable network solution.

\section{ACKNOWLEDGMENTS}

The authors wish to thank their parents for supporting and motivating for this work.

\section{REFERENCES}

[1] Ahmad Anzar, Joshi Pankaj, Rathi Vikas, Bagwari Ashish (IEEE Member), "A Cluster Head Gateway Approach for Deciding the Cluster head in Mobile Adhoc Network" Singapore Conference December 2011 ICCNS 2010: I"International Conference on Computer Networks and Securityl". 
[2] A. Amis, and R. Prakash, March 2000. Load- Balancing Clusters in Wireless Ad Hoc Networks. In Proceedings of the 3rd IEEE Symposium on Application-Specific Systems and Software Engineering Technology (ASSET'00), pp 25.

[3] D. J. Baker, and A. Ephremides, November 1981. The Architectural Organization of a Mobile Radio Network via a Distributed Algorithm. IEEE Transactions on Communications, COM-29(11): pp 1694-1701.

[4] J. Broch, D. Maltz, D. Johnson, Y. Hu, and J. Jetcheva, October 1998. A performance comparison of multi-hop wireless ad hoc routing protocols. In Proceedings of the Fourth Annual ACM/IEEE International Conference on Mobile Computing and Networking.

[5] M. Denko, 2003The Use of Mobile Agents for Clustering in Mobile Ad Hoc Networks, In Proceedings of ACM International Conference, Vol. 47, pp 241 - 247.

[6] Z. J. Haas, and M. R. Perlman, The performance of query control schemes for the zone routing protocol. In Proceedings of ACM Sigcomm'98, Vol. 28, No. 4, pp 167 - 177, October 1998. J. Computer Sci., 2 (7): 583-588, 2006. 588.

[7] Z. J. Haas, and M. R. Pearlman, 2000. "The zone routing protocol: A hybrid framework for routing in ad hoc networks," in Ad Hoc Networks, C. E. Perkins, Ed., Chapter 7, Addison-Wesley.

[8] I. Er, and W. Seah, July 2005. Clustering Overhead and Convergence Time Analysis of the Mobility-Based MultiHop Clustering Algorithm for Mobile Ad Hoc Networks, ICPADS, the 11th International Conference on Parallel and Distributed Systems.
[9] I. Er, and W. Seah, March 2004. Mobility-based d-Hop Clustering Algorithm for Mobile Ad Hoc Networks. In Proceedings of WCNC'04,

[10] M. Gerla, and J. Tsai, 1995. Multicluster, Mobile, Multimedia Radio Network. ACM Journal on Wireless Networks, Vol. 1, No. 3, pp 255-265,

[11] A. McDonald, T. F. Znati, Aug. 1999. A mobility based framework for adaptive clustering in wireless ad hoc networks. IEEE JSAC, 17(8):1466- 1486,

[12] F. G. Nocetti, J. S. Gonzalez, and I. Stojmenovic, 2003. Connectivity Based k-Hop Clustering in Wireless Networks, Telecommunication Systems Journal, Vol. 22, No 1-4, pp 205-220.

[13] S. Sivavakeesar, and G. Pavlou, Stable Clustering Through Mobility Prediction for Large-Scale Multihop Intelligent Ad Hoc Networks, In Proceedings of the IEEE Wireless Communications and Networking Conference (WCNC'04), Georgia, USA, Mar. 2004, vol. 3, 1488 -- 1493.

[14] Y. Wang, W. Wang, X. Li, 2005. Distributed Low-Cost Backbone Formation for Wireless Ad Hoc Networks, In the Proceedings of the 6th ACM international symposium on Mobile ad hoc networking and computing, pp $2-13$,

[15] A. Amis, P. Prakash, D. Huynh, and T. Vuong, Max-Min D-Cluster Formation in Wireless Ad Hoc Networks, Infocom 2000, The Conference on Computer Communications, Volume 1, pp 32-41., Nineteenth Annual Joint Conference of the IEEE Computer and Communications Societies. 